Cahiers d'études africaines

189-190|2008

Territoires sorciers

\title{
Déchirures et rumeurs
}

La chasse au sorcier et l'héritage idéologique de la révolution socialiste au Mozambique (Muidumbe, 2002-2003)

Paolo Israel

\section{OpenEdition}

Journals

Édition électronique

URL : https://journals.openedition.org/etudesafricaines/10472

DOI : 10.4000/etudesafricaines. 10472

ISSN : $1777-5353$

Éditeur

Éditions de l'EHESS

\section{Édition imprimée}

Date de publication : 7 avril 2008

Pagination : 209-236

ISBN : 978-2-7132-2141-5

ISSN : 0008-0055

\section{Référence électronique}

Paolo Israel, « Déchirures et rumeurs », Cahiers d'études africaines [En ligne], 189-190 | 2008, mis en ligne le 08 avril 2011, consulté le 04 mai 2021. URL : http://journals.openedition.org/etudesafricaines/ 10472 ; DOI : https://doi.org/10.4000/etudesafricaines.10472 


\title{
Paolo Israel
}

\section{Déchirures et rumeurs}

\author{
La chasse au sorcier et l'héritage idéologique \\ de la révolution socialiste au Mozambique \\ (Muidumbe, 2002-2003)
}

En 2002, dix ans après la fin de la guerre civile (1977-1992), les médias mozambicains rapportaient des histoires saugrenues, grotesques et mystérieuses provenant de Muidumbe, district de l'extrême nord du pays et berceau de la lutte de libération nationale ${ }^{1}$. Les récits parlaient de «lions fabriqués » ravageant les villageois et de foules enragées lynchant à mort les «fabricants de lions». La presse prenait parfois un ton condescendant et ironique envers «le peuple » superstitieux et illettré, se laissant parfois emporter par la fascination pour la puissance secrète de la «magie noire africaine ». Certains médias s'adonnaient aussi à des interprétations politiques. Le journal gouvernemental Notícias rapporta que les villageois de Muidumbe avaient entrepris de lyncher l'administrateur du district, parce qu'il ne les protégeait pas des «lions de la RENAMO ». Une émission radio, produite localement (Limbombo 2003) et diffusée à plusieurs reprises par la radio nationale, dressait un tableau dramatique conjuguant insécurité, mécontentement et conflit.

1. Le Frelimo (Frente de Libertação de Moçambique, Front de libération du Mozambique) fondé à Dar-Es-Salaam en 1962, déclencha une lutte de guérilla contre les Portugais à partir de 1964. Pour des raisons stratégiques, la guérilla eut lieu principalement dans les zones septentrionales du Mozambique, près de la frontière avec la Tanzanie, et notamment en pays makonde. En 1975, Samora Moises Machel levait le drapeau du Mozambique indépendant et le Frelimo devenait le parti unique marxiste-léniniste de la République populaire du Mozambique. Une guerre civile, parrainée par les gouvernements ségrégationnistes de la région (Afrique du Sud, Rhodésie), ne tarda pas à éclater (1977). Le mouvement de guérilla anti-gouvernementale, RENAMO (Resistência nacional moçambicana, Résistance nationale mozambicaine) fut bien accueilli parmi certaines composantes de la population mozambicaine, déçues des politiques draconiennes du parti unique. Après quinze ans de guerre civile (1977-1992), le pays connaît la paix. Le FrELIMO continue à gouverner, après avoir gagné trois fois de suite $(1994,1999,2004)$ les élections. 
Les événements de Muidumbe se prêtent bien à une lecture politique qui appréhende la sorcellerie africaine contemporaine en tant que discours (et pratique) de pouvoir lié aux crises de la modernité et de l'État postcolonial $^{2}$. Certaines facettes - notamment la prépondérance des jeunes dans les lynchages et les rumeurs de vampirisme et de trafic d'organes - renvoient à des phénomènes globaux qui ont été mis en relation avec l'émergence et la brutalité du capitalisme du nouveau millénaire (Comaroff \& Comaroff 1999, 2001). S'attacher d'emblée à ces suggestions globalisantes peut conduire pourtant à se fourvoyer. Muidumbe est autant une périphérie économique exposée aux fantômes «aliens » du néo-libéralisme (Comaroff \& Comaroff 2002), que le centre longuement idéalisé du projet de révolution moderniste du Frelimo. Les faits de violence et de sorcellerie qui y eurent lieu en 2002-2003 présentent une cohérence narrative et symbolique, qui autorise leur appréhension comme une «crise » obéissant à une logique unitaire. Dans la lecture que j'en donnerai, cette crise renvoie à l'articulation locale du projet révolutionnaire de construction de la nation mozambicaine, à la formation historique des subjectivités de ses pionniers, et notamment à l'incorporation de dispositifs idéologiques paradoxaux ${ }^{3}$. Pour soutenir cet argument, seront aussi interrogées des pratiques performatives locales (notamment la danse des masques mapiko) qui mettent en scène — toujours de manière ambiguë — la constitution de ces subjectivités, aussi bien qu'elles participent à leur formation.

\section{Muidumbe, 2002-2003 : la crise des lions}

La veille de Pâques 2003, le district de Muidumbe était sur le point d'imploser. Tout avait commencé la nuit du 8 juillet 2002, quand un «lion » avait

2. Pour un survol de la littérature produite par l'école de la «modernité de la sorcellerie », voir entre autres Ashforth (2005 : 116-117), GeschiERE (2000), MoOre \& SANDERS (2001: 10-13), et pour une lecture critique, SANDERS (2004).

3. Les analyses qui suivent se basent sur des recherches de terrain à Muidumbe (Mozambique, Cabo Delgado, 2002-2006). Une partie du travail de terrain a été conduite en collaboration avec Estevão J. Mpalume de la Direction provinciale de la Culture de Cabo Delgado. 61 entretiens ont été réalisés auprès des protagonistes de la crise (victimes des attaques, accusés de sorcellerie, guérisseurs, chasseurs, fonctionnaires de l'administration publique, autorités communautaires, etc.). Je me suis attaché dans un autre article (IsRAEL à paraître) à une analyse de la crise de Muidumbe, qui prend en compte plutôt les facteurs microsociaux et politiques, les données écologiques, ainsi que l'histoire des pratiques de la violence.

4. Chacune des provinces du Mozambique est divisée en districts, les districts en postes administratifs et en localités. L'autorité principale d'un district est son administrateur, qui répond au gouverneur de la province. Dans le district de Muidumbe (province de Cabo Delgado, dont la capitale est Pemba) les populations vivent dans des villages communaux, résidus du projet révolutionnaire, de 1000 à 10000 habitants (WEST 1998). 
tué quatre personnes dans les «proches plaines » de Ing'unde, en dessous de la mission de Nangololo ${ }^{5}$. La «bête » avait suivi le sentier accidenté qui côtoie le fleuve saisonnier, et avait attaqué quatre fois dans cette zone de production agricole, allant parfois jusqu'à dénicher ses victimes dans les huttes précaires construites dans les champs. Par quatre fois, elle avait été dérangée par des voisins, l'empêchant de consommer la viande qu'elle avait chassée. La cinquième fois, elle captura sa victime et l'emmena avec elle au fond de la brousse.

Sur son chemin, le «lion» avait laissé un blessé. L'homme — un Makua $^{6}$ de Chiure qui était en train de fabriquer de l'eau-de-vie de canne à sucre avec un compagnon ${ }^{7}$ — avait survécu à l'attaque. Le matin suivant, il fut emmené à l'hôpital de Mwatide. Diagnostic: blessures d'origine inconnue, profondes et nettes, suspectées provenir d'une arme perforante. Une fois soigné, l'homme raconta aux médias locaux une histoire fort bizarre. Le «lion » qui l'avait attaqué n'était pas un lion : il s'agissait d'un homme, qui s'était déguisé en lion avec des peaux, et qui l'avait poignardé. La victime identifia son assaillant : il s'agissait du frère de son compagnon, un jeune ivrogne «bon à rien ». Quelques jours plus tard, une foule de jeunes hommes enragés enlevèrent le «lion» de sa maison au village de Mwambula (où vivaient tous les protagonistes de cette histoire), l'emmenèrent sur le chemin qui conduit vers le village de Namaande, et le tuèrent à coups de machettes et de bâtons. Son corps fut éventré, et laissé sans sépulture pendant un jour. La tuerie eut lieu à quelques centaines de mètres de la maison de l'administrateur du district.

Ce cas fut le premier d'une longue série. Dans les proches plaines des villages de Mwambula, Namakande, Namakule, Nshinga, Nang'unde — et dans tous les villages des plaines du fleuve Messalo - les «lions » attaquaient les hommes, souvent ceux habitant dans les zones de production ou aux bords des villages, qui sortaient de nuit de leurs paillotes. Les cadavres étaient découverts dans les zones de brousse souvent à moitié dévorés. Un bras ou une jambe pouvaient aussi être les seuls indices de la tuerie. Après l'homme Makua, d'autres rescapés racontaient des histoires

5. L'écologie du plateau makonde ne permet pas que ses terres donnent à ses habitants tous leurs moyens de subsistance. Chaque village est dépendant d'une plaine pour plusieurs activités agricoles, et surtout pour le ravitaillement en eau. Á côté de ces plaines de proximité, il existe des plaines plus lointaines qui entourent le plateau aux quatre points cardinaux. Les grandes plaines méridionales prennent leur nom du fleuve qui côtoie le plateau, le Messalo. (Ku-)Ingunde est le nom des plaines de proximité qui ravitaillent plusieurs des villages du plateau, proches de ses pentes sud-orientales.

6. Ethnonyme.

7. La préparation et la consommation de boissons distillées eurent une certaine influence sur le développement de la crise (ISRAEL à paraître). Ing'unde est une zone de production de canne à sucre et de distillation. Les lions attaquaient souvent des personnes saoules, ce qui jetait bien évidemment des doutes sur la véracité des récits des rescapés. 
saugrenues : des lions qui frappaient avec des marteaux; des hommes avec des couteaux, déguisés avec des peaux et des masques; des lions qui attaquaient et parlaient ensuite; des empreintes de pattes de lion se transformant en traces de tongs après quelques centaines de mètres; des hommes qui étaient tués par des «lions » qui leur enlevaient leur chemise...

Les hommes répondaient en tuant des «lions $»^{8}:$ des sorciers (vavi) suspectés de se transformer (kupika) en lion. La désignation des suspects (kutambulanga uwavi) avait lieu tantôt suivant les règles traditionnelles du conseil familial ou de la divination (yangele), tantôt selon une logique tout à fait nouvelle. Des indices circonstanciels pouvaient accuser l'un ou l'autre: être en possession de peaux, avoir sa paillote encerclée par des traces de pattes de lion, se trouver au mauvais endroit au mauvais moment, étaient des raisons suffisantes pour révéler en quelqu'un un «lion », et pour l'exposer à la rage meurtrière des lyncheurs.

Les rumeurs couraient plus rapidement que les bêtes. "Les tueries sont l'œuvre d'une bande d'assassins qui se déguisent en lion, à l'aide de peaux et de pattes de lions sculptées. Les lions font leurs entraînements (likulutu) dans les plaines, où ils ont une base d'opération. Les lions cherchent à acquérir des organes humains pour les vendre, séchés, à des guérisseurs, en Tanzanie. L'administrateur est le chef des lions. Il a vendu le district aux Blancs. Sur le mont Ing'ombe, au centre des plaines de Ing'unde, on peut voir un drapeau du Frelimo renversé : c'est l'étendard des lions. L'administrateur vole toutes les nuits dans un hélicoptère magique pour rejoindre le mont Ing'ombe... »'.

Déconcertés face à l'action de ces nouveaux «lions transformés » ( «vantumi vakuvapika») — qui n'étaient pas évoqués à l'aide des techniques traditionnelles ${ }^{10}$, mais se promenaient armés de couteaux —, les guérisseurs répondaient aux questions de façon hiératique : "Nangu namanya shinu » («moi j'en sais rien »). Les chasseurs, mobilisés par le gouvernement local pour tuer les lions, se montrèrent réticents, et — craignant la confrontation

8. Pour éviter de prendre parti sur la question épineuse de la rationalité de la sorcellerie (ASHFORTH 2005 : 113-116; WeSt 2007 : 19-25), j'utilise la terminologie ambiguë employée par les protagonistes de la crise. Était appelé «lion » tout être (animal ou humain) identifié comme l'agent d'une tuerie dont les indices externes montraient l'action d'un lion, autant « de brousse » ( «wakumwitu ») que «fabriqué » («wakuvapika»): blessures dérivantes de griffes, démembrements, morsures, ainsi que blessures perforantes...

9. À noter la prépondérance d'un imaginaire moderniste et notamment militaire (hélicoptères, mines, fusées) dans les métaphores de sorcellerie en shimakonde (langue Makonde).

10. Les guérisseurs affirment que la transformation d'homme en animal (lion, hyène, éléphant, etc.) se fait par l'intermédiaire de petits bouts de bois appelés dimika, qui aident le sorcier à se transformer en animal, ou à évoquer un animal qu'il contrôlerait ensuite. Les blessures infligées au lion fabriqué (kupika) se transfèrent sur le sorcier (kulibyaa uwavi: se blesser soi-même de sorcellerie). 
avec des animaux magiques et donc tenus pour invulnérables - usaient plus volontiers les fusils pour abattre des animaux comestibles.

Au cours de la saison des pluies, la situation empira. Les « lions » continuaient à attaquer hommes et femmes dans les plaines et les villages, et les foules tuaient davantage. Le district entier tomba en proie à la peur et au mécontentement politique, dirigé contre le gouvernement qui non seulement ne faisait rien contre les « lions », mais essayait d'empêcher la population de les punir en les «fusillant» (lynchant). L'administrateur était le principal accusé, suspecté par la population locale d'être le chef des lions. Incapable de résoudre cette crise, il se défendait en invoquant le manque d'appui du gouvernement provincial, qui n'envoyait pas d'armes ou de brigade de chasseurs. Les présidents des villages ${ }^{11}$ durent prendre parti - pour le gouvernement et sa vision rationaliste, ou pour les foules de lyncheurs. La plupart prirent une attitude à la Ponce Pilate. Certains, au péril de leur vie, interdirent les lynchages. D'autres, le plus souvent poussés par des intérêts politiques et/ou personnels, commandèrent aux milices populaires sur leurs ordres (grupo doze) d'exécuter certains des «lions».

En avril 2003, les «lions » avaient tué cinquante-cinq personnes, et les lyncheurs mis à mort vingt-quatre « lions » — à l'apparence bien humaine et aucun lion ne ressemblant à un lion ${ }^{12}$.

La nuit de Pâques, un lion enleva un jeune homme aux abords du village de Mwambula. Une foule guidée par un guérisseur du village de Mwatide et par le vice-président du village, suivit ses empreintes sur plus de vingt kilomètres dans la brousse des plaines de Ing'unde. Elles se terminaient dans la hutte de brousse d'un homme qui fut identifié comme un habitant de Namakande. Le jour suivant, un escadron de jeunes des villages de Mwambula, Nshongwe et Namakande enlevèrent le «lion » de sa maison, et l'emmenèrent à Mwambula pour le «fusiller» publiquement.

Un jeune homme, de Mwambula, la vingtaine, prit les choses en main. Il monta sur le podium destiné aux autorités et prononça devant la foule un plaidoyer pour que les jeunes prennent le devant de la vie politique, contre le gouvernement. «Si le Frelimo ne nous défend pas des lions, à partir d'aujourd'hui, le Frelimo c'est nous. » Une croix de bois fut apposée

11. Dans les zones libérées du contrôle des Portugais, le Frelimo remplaça les « autorités gentilices » («autoridades gentilicas ») qui fonctionnaient comme interface dans le système colonial portugais, par des structures du Front (ADAM 1993). Sur le plateau makonde, les figures d'autorités claniques continuèrent pourtant à exercer un rôle important dans la résolution des conflits sociaux (WEST 1998). Depuis 1975, les villages communaux en pays makonde sont gouvernés par des structures locales élues avec un suffrage démocratique (président, vice-président, assesseurs, chefs des quartiers). Leur autorité se fonde souvent sur un prestige de type clanique, ou sur le support d'un conseil d'anciens.

12. Plusieurs lions à l'apparence de lion avaient été tués avant le début de la crise dans les villages du plateau les plus proches des plaines. Certains guérisseurs affirmaient pourtant qu'il ne s'agissait pas de lions normaux. 
sur le front $\mathrm{du}$ «lion ». La foule entonna ce qui était depuis quelques mois l'«hymne » des lyncheurs :

$\begin{array}{ll}\text { Vashikaji noma } & \text { Jeunes «pantins » } \\ e-e-e & \text { e-e-e } \\ o-O-O & \text { O-O-O }\end{array}$

suivi d'une «chanson » spécialement « composée » pour l'occasion :

Wenda kwashi baba

Nelo wenda kwashi?

«Namanya shinu»

Wenda ku-Kalavali nelo!
Où vas-tu, père

Aujourd'hui, où vas-tu?

«Je ne sais pas»

Aujourd'hui, tu vas au Calvaire!

Le «lion» fut emmené sur le chemin de Namaande, et tué.

\section{Lions locaux, rumeurs globales}

Suite à cette ébauche d'émeute, le gouverneur de la province envoya une brigade de chasseurs qui tuèrent un lion — gros, vieux et claudicant — aux abords du village de Malangonya. Celui qui avait abattu le lion était pourtant convaincu que les faits de Muidumbe n'étaient pas exclusivement l'œuvre des bêtes sauvages.

« À Muidumbe j'ai vu des choses louches. Ils m'ont montré des traces de lion... Moi je dis que ce n'était pas un lion. Le président du village de M. se promenait toujours avec une femme... Il aurait fallu l'interroger celle-là. Je pense qu'elle en savait beaucoup. Et après coup, il est parti en Tanzanie avec un panier d'escargots. Tu ne comprends pas ? Ça a la même odeur des organes séchés ! Il faut interroger cette femme. Autrement, d'ici un mois, quand le brouhaha se sera tu, ces gens vont recommencer à tuer à nouveau. »

Les prévisions du chasseur - neveu d'un chasseur portugais renommé à l'époque coloniale (West 2007 : 21-22, à paraître) — ne se réalisèrent pas. Après la mort du gros lion, connu dans la zone comme «le balayeur» («kumpyaya»), la crise se termina soudainement ${ }^{13}$. Dans les mois qui suivirent, plusieurs lions furent tués par des chasseurs locaux, et l'on ne parla plus de la «guerre des lions » que comme un événement aussi tragique que révolu.

Les histoires (et les notions) d'hommes se transformant en lions et autres bêtes sauvages sont anciennes et enracinées en pays makonde (West 2005 : 45-46 et sq.), et sont d'ailleurs répandues ailleurs en Afrique (Jackson 1990).

13. Les lions ne cessèrent pas pour autant de se nourrir sporadiquement de chair humaine dans les districts environnant Muidumbe, sans donner lieu à aucune crise. Notamment en 2004, un lion dévora plusieurs personnes dans les districts de Mocimboa da Praia et Palma. 
La crise de 2002-2003 marqua pourtant l'avènement d'un nouveau paradigme local de la sorcellerie, notamment par deux aspects. Tout d'abord, les modalités de la transformation et de l'action des sorciers s'en trouvaient changées. Les lions de Muidumbe agissaient en groupe, se déguisant à l'aide de peaux, de griffes et de pattes sculptées, comme l'avaient fait certaines sociétés secrètes ailleurs en Afrique à l'époque coloniale. Tout en restant conceptualisée comme une transformation magique (kupika), la métamorphose des sorciers ne se basait plus sur un savoir-faire ésotérique - celui des dimika - mais sur une technique de fabrication et sur un entraînement collectif (likulutu). Ensuite, les motivations des «lions» étaient aussi bien nouvelles. Ils ne se limitaient plus à saisir (kunkamula shintumi) les membres de leur famille. Ils attaquaient de façon apparemment arbitraire, parfois s'en prenant même à des inconnus ou à des forestiers. Leur motivation n'était pas nécessairement l'assouvissement d'une faim insatiable, ou l'envie (ing'ou) ${ }^{14}$, mais le prélèvement d'organes, à vendre séchés sur le marché international de la médecine traditionnelle. L'apparition de ce nouveau paradigme local déconcerta les spécialistes locaux de l'anti-sorcellerie eux-mêmes. Face à ces nouvelles donnes, les guérisseurs déclaraient leur impuissance : «De ces lions qui tuent avec des couteaux, on n'en sait rien ${ }^{15}$. Si la sorcellerie est un langage de pouvoir (Kapferer 1997 : 263-274; West 2005 : 2-8), ses locuteurs ne semblaient plus en maîtriser la grammaire.

Ce changement de paradigme inscrit la crise de Muidumbe dans des processus sociaux plus larges, renvoyant aux transformations induites à l'échelle globale par le capitalisme du nouveau millénaire (Comaroff \& Comaroff 2001), et puisant dans un imaginaire d'aliénation de la longue durée (Shaw 1997). Les rumeurs globales concernant le trafic d'organes (Scheper-Hugues 1996, 2000) ont été mises en relation avec la violence symbolique coloniale et postcoloniale (White 1997) ou bien avec la dépossession des corps liée à la rapacité du capitalisme (les organes seraient «a materialized form of cultural capital » Comaroff \& Comaroff 1999 : 286). Il est plus difficile de comprendre pourquoi lors de la crise de Muidumbe ressurgirent des fantômes provenant d'autres temps et d'autres coins d'Afrique. Nul n'a prouvé l'existence d'une société secrète d'hommes-lions à Muidumbe, sur le mode de celles documentées ailleurs en Afrique pendant la période coloniale (Joset 1955 ; Nwaka 1986), se déguisant à l'aide de peaux et pattes et tuant à des fins rituelles, mais aussi politiques (Rich 2001). Pourquoi le changement local de paradigme de la sorcellerie de Muidumbe s'articula autour de cet imaginaire colonial ? Comment les faits se tournèrent en représentations et traversèrent les espaces et les temps ?

14. Voir ultra pour une définition plus précise.

15. Le guérisseur le plus connu pour savoir des choses au sujet des lions se refusa à participer à la chasse collective qui eut lieu lors de la dernière attaque du lion. Pourtant une fois le lion tué, ils recommencèrent à appuyer les chasseurs avec des techniques pour emprisonner les lions. 
La facilité avec laquelle ces rumeurs globales prirent pied à Muidumbe témoigne tout au moins du fait que l' «insécurité spirituelle » (Ashforth 2005 : 3) de cette partie du Mozambique n'est pas foncièrement différente de celle d'autres endroits de la planète, dans l'articulation de ses cauchemars. Considérer les rumeurs liées à la crise de Muidumbe comme la matérialisation de spectres de dépossession - récents aussi bien que de longue durée - est tout à fait légitime. Le plateau makonde est inséré dans des réseaux internationaux économiques asymétriques et d'exploitation brutale, au moins depuis qu'il porte le nom de ses habitants ${ }^{16}$. Plus récemment, le tournant néo-libéral se matérialise pour les habitants de Muidumbe sous différents visages - très concrètement, ceux des coupeurs de bois qui ravagent les plaines du fleuve Messalo, et dont l'action pourrait expliquer les mouvements des populations de lions.

La crise de Muidumbe ne fut pas non plus un cas complètement isolé au Mozambique. Des explosions de violence populaire s'appuyant sur des rumeurs globales — notamment concernant le choléra — eurent lieu au cours de l'année 2002 en d'autres districts du nord du Mozambique, et aussi dans un village des plaines de Muidumbe. À l'occasion d'une épidémie de choléra, dans la province de Nampula en 2002, des rumeurs d'empoisonnement des puits d'eau par certaines ONG aboutirent à des épisodes de lynchage (Serra 2003b). Dans la même année, à Muidumbe, des incidents similaires se reproduisirent, soutenus par des rumeurs semblables (concernant pourtant l'État et non pas l'action des ONG, qui y est presque inexistante). Dans le village de Myangalewa, des centres de traitement du choléra (CTC) furent incendiés par la population. Le gouvernement n'intervint point, et la crise s'étouffa toute seule une fois que les guérisseurs défendant la nature magique de l'épidémie commencèrent à mourir du choléra un à un. Ces crises locales furent aussi médiatisées en tant que phénomènes politiques, et parfois instrumentalisées par le gouvernement ou par l'opposition. Pourtant, aucun de ces cas de révolte ponctuelle n'atteignit la gravité, le niveau de violence et la densité symbolique de la crise des lions de Muidumbe ${ }^{17}$.

Au-delà des idiosyncrasies des cas de lynchage, la crise de Muidumbe présente une « unité narrative » (Ricœur 1985) tout à fait remarquable. De par ce fait, elle fut qualifiée de «guerre » («ing'ondo yavantumi », la guerre des lions), ayant un événement déclencheur, une longue phase croissante d'altercations, et un apex dramatique. Il faut aussi mettre en avant la localisation des événements — dans un seul district - et l'investissement politique des rumeurs et de l'interprétation collective de la crise.

16. Notamment : la traite esclavagiste, l'exploitation de la part de compagnies à charte à capitaux internationaux, l'étatisation de la part du pouvoir colonial salazariste.

17. Ailleurs au Mozambique pendant la période coloniale, on enregistre des crises de chasse au sorcier aussi graves que celle de Muidumbe, probablement soustendues par des logiques différentes. Pour le cas de la Zambézia, voir SERRA (2003a: 48, 150). 
Il faut aussi considérer les inversions symboliques tout à fait paradoxales qui marquaient les lynchages. La dernière victime fut transfigurée en image christique, juste le jour après Pâques. Les bourreaux se condamnaient euxmêmes, par l'imagerie qu'ils avaient adoptée, au pire des crimes. La terminologie indiquant les lynchages était en elle-même contradictoire. "Kutannola », traduit en portugais comme fuzilar (fusiller) était un verbe utilisé à l'époque de la lutte de libération pour indiquer les lynchages populaires des contrerévolutionnaires. Toutefois, à l'origine, le verbe était lié à une pratique de sorcellerie qui consisterait à sucer le sang d'une victime, comme un vampire (ce qui évoque une imagerie bien ancienne en Afrique, liée au colonialisme et à la modernité) (Serra 2003a : 46-54; White 1993, 2000). Les lyncheurs auraient été donc eux-mêmes des sorciers/vampires ? Une fusillade — et non des paroles - mit fin à la crise. Rétrospectivement, elle fut considérée comme une attaque de folie collective. Ceux qui avaient tué nièrent. Ceux qui avaient crié contre le gouvernement hochèrent la tête : « À cette époque, on était devenu tous un peu fous...»

Qu'est-ce qu'avait provoqué cette attaque de folie exclusivement chez les villageois de Muidumbe ? Pourquoi s'étaient-ils tellement enragés contre leur gouvernement local ? Pourquoi les lynchages collectifs guidés par des jeunes prenaient place aussi dans les villages du plateau relativement protégés des incursions des lions ? Pourquoi disait-on qu'un drapeau renversé du Frelimo était planté sur l'endroit mystérieux où se seraient entraînés les membres de la société secrète d'hommes-lions ? Qui étaient-ils les «jeunes pantins » dont les méfaits auraient poussé les jeunes lyncheurs de Muidumbe à mettre en scène une révolte aussi improbable que grotesque ?

La crise de Muidumbe reste illisible, si elle n'est pas appréhendée dans le cadre de dynamiques globales et de la longue durée, de dépossession et d'insécurité. Cependant, les raisons de son exacerbation doivent à mon sens se chercher ailleurs — non seulement en prenant en compte les spécificités politiques, culturelles et microsociales de la crise, mais en s'attachant à la structuration historique des subjectivités de ceux qui en furent les acteurs.

\section{«Doubles contraintes » de la révolution}

Le gouvernement local, et notamment l'administrateur du district, justifiait les faits de Muidumbe en s'appuyant sur une donnée principale : le taux d'analphabétisme de ses habitants.

«Qu'est-ce que vous pouvez attendre d'une population qui a un taux d'analphabétisme de 80-90\% ? Les lions sauvages mangent les gens. Les gens, à cause de leur ignorance, s'entre-tuent. Il y a beaucoup d'opportunistes qui utilisent l'histoire des lions pour régler des comptes. Mais la plupart sont des ignorants qui n'envoient pas leurs enfants à l'école. C'est aussi simple que ça. Il est scientifiquement prouvé qu'un homme ne peut pas se transformer en lion. Point barre. La raison de ces événements est la superstition, causée par l'analphabétisme. » 
Pour l'administrateur de Muidumbe, son peuple était foncièrement prémoderne, enferré dans les pièges d'une tradition que la technologie, la science et le progrès auraient inéluctablement effacée. Des discours de ce type - qu'il tenait dans toutes les instances officielles, aussi bien que lors de longs défoulements dans l'intimité de sa maison ${ }^{18}$ — étaient loin d'être nouveaux à Muidumbe, au pays makonde, et au Mozambique en général. Il s'agissait de l'idéologie du socialisme scientifique du FreLIMo de la première heure, de la «page blanche » et de l' «homme nouveau » libéré des lourdes chaînes de la tradition ${ }^{19}$. Les nuances du discours international apparu récemment à Maputo, valorisant la connaissance indigène et l'autorité traditionnelle, étaient bien loin d'effleurer Muidumbe, berceau et sanctuaire de la révolution.

Au-delà de l'analphabétisme, l'administrateur, le gouvernement et la presse avaient recours aux clichés ethniques sur les Makonde (notamment ceux de Muidumbe) : non civilisés, violents et insoumis ${ }^{20}$.

« Ici à Muidumbe, les gens sont compliqués. Ici, c'est le cœur des Makonde. Pour cette raison, il faut toujours intervenir après coup. Si sur le moment tu t'interposes entre deux personnes qui se bagarrent, c'est à toi qu'ils vont s'en prendre. »

Lors de la crise de 2002-2003, les stratégies habituelles qui lui avaient permis de survivre longtemps comme administrateur ${ }^{21}$ ne fonctionnaient plus. Plus il laissait faire, plus les gens tuaient. Plus il défendait une idéologie rationaliste, plus les gens enrageaient contre lui. Le jour de Pâques, après

18. En même temps, la crise avait exacerbé son modernisme, le repoussant vers un radicalisme idéologique qu'il n'avait jamais connu. Quelques mois avant le début des événements - avant qu'il ne puisse plus se promener dans sa propre terre sans être pointé du doigt par les enfants qui criaient auprès de sa voiture : ntumi! ntumi! (lion! lion !) - il tenait des propos fort différents. Il racontait comment Samora Machel l'avait enlevé de son poste d'administrateur à Mambone après seulement trois jours de travail, en lui disant : «Ces Ndau vont te tuer avec leur sorcellerie »; il racontait comment il avait été emmené par des employés d'une administration passer un rituel où il avait vu des dizaines de serpents obéir à une femme nue ; il racontait des histoires du temps de guerre, d'hommes ensorcelés qui étaient obligés d'uriner du fil à coudre pendant des jours... À partir du moment où il connut le poids de la rumeur, il ne raconta jamais plus une de ces histoires.

19. La critique de l'idéologie révolutionnaire du Frelimo par Christian GefFray (1988, 1990) reste à ce jour une référence incontournable, voir aussi CAHEN (1987), Hall \& Young (1997).

20. L' «attitude d'insoumission » est une constante dans la construction de l'identité makonde, qui a été déclinée sous plusieurs formes dans des circonstances historiques différentes (ADAM 1993: 1) AsKEW 2002 : 213). Le stéréotype est présent depuis les premiers récits d'explorateurs (WEST 2004), et se nourrit ensuite de l'histoire de la résistance des Makonde au colonialisme et de la participation à la lutte de libération. La rhétorique anticoloniale de la lutte s'est plaquée sur ce mythe, l'a amplifié et l'a transformé. Le factuel et l'imaginaire se soutiennent réciproquement dans la constitution de ces configurations identitaires.

21. Au sens littéral. L'administrateur précédent avait été tué par des villageois. 
l'assassinat du «lion » de Namakande, il envoya un message de détresse au Gouverneur, déclarant son impuissance. « La situation est dramatique avec des conséquences imprévisibles. »

Une double contrainte (double bind) est une situation d'interaction interpersonnelle dans laquelle une injonction négative primaire explicite entre en conflit avec une injonction secondaire implicite, qui se situe à un niveau plus abstrait (Bateson 2000 : 206-207). Les deux injonctions sont accompagnées par des menaces de punition, au cas où elles seraient contrevenues. Les doubles contraintes ont la caractéristique de ne pas pouvoir se résoudre rationnellement. Plus elles sont investies du point de vue émotionnel, plus elles sont efficaces et paralysantes. L'école de Palo Alto identifie les situations d'exposition aux doubles contraintes comme source de syndromes schizoïdes et de troubles dans la métaphorisation.

Pour une interaction complexe de facteurs stratégiques, historiques et circonstanciels, les Makonde s'engagèrent dans le projet de la lutte de libération (Adam 1993 ; West 2005 ; Israel 2008) suivi de la militarisation de la société pendant dix ans ; la mise en place des politiques socialistes (villages communaux, production collective, etc.) ; l'implantation efficace de la machine administrative du parti et l'idéalisation du pays makonde comme berceau de la révolution et laboratoire de la construction de l' « homme nouveau » ( «homem novo ») révolutionnaire. La culture, la société et l'identité makonde se redéfinirent et se re-modelèrent autour du FrELIMO, par un processus d'incorporation de l'idéologie révolutionnaire. Par un mouvement de vernacularisation, le discours internationaliste du parti devint le pilier central autour duquel se reconstruisit une identité locale et particulariste.

La révolution du FRELIMO aboutit à l'incorporation de dispositifs disciplinaires (au sens foucaldien) basés sur des doubles contraintes ${ }^{22}$. Ce processus de subjectivation (Butler 1997) est mis en route non seulement par des dispositions imposées du haut, par le parti, mais par une «tension conviviale » (Mbembe 2000b : 142) entre «les chefs » et «le peuple ». L'injonction négative primaire explicite dérive directement des dispositions et des dispositifs idéologiques provenant de la direction centrale du parti (disons, de l'idéologie officielle). Elle est renforcée par des pratiques coercitives publiques et humiliantes comme la flagellation; par la marginalisation sociale (apposition de labels : réactionnaire, ennemi du peuple) ou bien par la disparition physique (exécution publique ou déportation dans des champs de rééducation). L'injonction secondaire implicite découle, en revanche, d'interprétations locales de l'action et de l'idéologie du parti-État (donc de métadiscours locaux). Elle est en quelque sorte un négatif ou un renversement de l'idéologie officielle, une lecture à rebours. Cette injonction prend aussi la forme d'un «secret public»: ce que tout le monde sait, mais ne peut pas être dit (Taussig 1999). L'injonction implicite trouve son origine dans

22. WALKER (1989) applique spécifiquement la théorie de la « double contrainte » à l'idéologie marxiste-léniniste. 
le cadre de systèmes des valeurs, d'institutions, de «pratiques signifiantes » (Comaroff \& Comaroff 1993 : 37) et d' «expressions humaines significatives » (Mbembe 2000a: 16) qui — sans qu'ils puissent se conceptualiser suivant des paradigmes culturalistes ou ethniques — sont liés à des « espaces sociaux » (Condominas 1980) déterminés. Les promesses de punition associées à la deuxième injonction implicite sont aussi cachées, soupçonnées ou invisibles. Elles ne sont pas pour autant moins menaçantes.

Considérons deux exemples :

Première double contrainte : l'anti-tribalisme. L'idéologie et la pratique de la construction de la nation mozambicaine du Frelimo était basée sur la transformation radicale de la société, du mode de production et de vie, et de l'adhésion à une communauté nationale dans laquelle se dissoudrait toute identité locale. L'injonction idéologique primaire du parti condamnait explicitement le «tribalisme» (le particularisme ethnique). Ceux qui le pratiquaient étaient punis par l'humiliation ou la marginalisation. La deuxième injonction implicite (et paralysante) découlait du décryptage d'une pratique tribaliste cachée au cœur du Parti ${ }^{23}$. La guerre et le processus d'étatisation avaient créé de nouvelles hiérarchies sociales, et une échelle de valeurs se construisit sur la base du degré d'engagement dans la lutte de libération. Ceci était particulièrement vrai pour les Makonde qui, par leur participation massive à la lutte, s'étaient retrouvés projetés — de façon symbolique autant que réelle - au centre de la scène nationale. La participation à la lutte de libération se traduisait en capital qui se transférait aussi de façon lignagère. En pays makonde, le particularisme familial et ethnique se justifiait donc par une invocation du capital symbolique et social dérivé de la participation à la lutte de libération nationale. « Nous, qui avons fait la guerre... » « nous, qui avons libéré [le pays]. » La deuxième injonction implicite donc appelait à « être » (au sens performatif autant que généalogique) un de « ceux » qui avaient libéré le pays. Le parti distribuait des gratifications et des punitions selon des clivages ethniques ou familiers, consistant en capital symbolique, social (notamment la possibilité de s'insérer dans le système scolaire du FRELIMO) et financier ${ }^{24}$.

Deuxième double contrainte : l'anti-obscurantisme. L'idéologie moderniste du parti condamnait tout discours non scientifique comme obscurantiste et réactionnaire. Il sanctionnait les « obscurantistes » de la même façon que les «tribalistes ». Toutefois, le discours du pouvoir de la classe dirigeante mozambicaine, de façon similaire à ce qui pouvait se passer ailleurs sur le

23. En ligne générale, l'existence d'une «clique » du sud dans la direction du parti est objet de discussion jusqu'à aujourd'hui au Mozambique (CAHEN 1987, 2000 ; Ncomo 2003). Il est intéressant de noter que la thèse de doctorat en sociologie d'Eduardo Mondlane soit dédiée au conflit entre loyauté ethnique et loyauté nationale en temps de crise (NсOMO 2003).

24. L'attribution de pensions de guerre à Cabo Delgado passe aussi par des réseaux familiers et de connaissance, souvent sans tenir compte de l'engagement réel dans la lutte. 
continent (Bayart 1990 : 288-295 ; Geschiere 1995) employait des métaphores et des tropes puisés dans le langage du pouvoir de la sorcellerie. Le peuple était censé abandonner tout discours obscurantiste, mais leurs dirigeants manipulaient un pouvoir secret et invisible (injonction et menace implicite). Cette deuxième injonction implicite se construit à partir d'une lecture de l'action politique du FRELIMO en tant que sorcellerie constructive (uwavi wakudenga, sorcellerie de construction) (West 2005, 2007 : XI), enracinée dans un «discours de pouvoir» local (West 2005) ${ }^{25}$.

L'administrateur de Muidumbe avait été élu comme symbole de la crise des lions parce qu'il était l'incarnation de ces contradictions idéologiques de l'ordre social - ordre que les jeunes lyncheurs auraient symboliquement et concrètement attaqué avec leurs actions. Tout en restant connecté à une dimension locale, il avait « mangé la nation » (« kulya shilambo »), ses règles et ses codes. Son ventre le suggérait métaphoriquement. Sa gestion du pouvoir le montrait aussi : recevant les citoyens dans sa résidence privée, leur parlant en langue locale et les traitant selon les codes d'étiquette traditionnelle - les renvoyant, dans les discours, à leur position en termes d'allégeances claniques ( tu es le fils d'un tel et d'une telle») et de prestige politique («quand mon père s'est engagé dans la lutte de libération, ta famille était du côté des Portugais ») — il faisait pourtant recours à la langue de bois de la bureaucratie pour trancher les discussions et renforcer son autorité. Bien avant les événements de 2002, sa grande maison blanche au centre du village avait été l'objet de rumeurs de sorcellerie. La nuit, on disait que du sang coulait de ses murs. Selon certains, il s'agissait du sang des personnes vampirisées pour la construire, selon d'autres, du sang des sorciers qui s'y étaient battus. En même temps, il n'avait pas tardé à embrasser les nouvelles politiques néo-libérales promues par le FMI au Mozambique, en se lançant comme petit entrepreneur local, en s'appuyant sur les structures délabrées de l'État socialiste (West à paraître) ${ }^{26}$. Ce contraste entre esprit égalitaire (reformulé dans un langage socialiste) et individualisme (néolibéral) s'inscrit dans une logique plus générale des discours de sorcellerie en Afrique (Geschiere 1995).

L'intervention du gouvernement, basée sur l'attirail idéologique du FreLimo, renvoyait le blâme au peuple de Muidumbe, illettré (donc obscurantiste) et prisonnier de son caractère ethnique (donc tribaliste). Tout en

25. «L'histoire, finalement, participerait d'une grande économie de la sorcellerie. Chose que la langue vernaculaire reconnaît bel et bien, mais que le lexique marxiste empêche cependant de nommer comme tel » (MBEMBE 2000a : 26-27).

26. WEST (2005) analyse la sorcellerie en pays makonde en tant que «langage de pouvoir » et «genre discursif». Son analyse de la crise des lions (WEST 2005: 250-251, à paraître) met en relation cet événement avec le processus de démocratisation et de réforme néo-libéral au Mozambique. La réponse aux attaques des lions serait donc une sorte de commentaire des méfaits de la classe dirigeante postsocialiste, qui, au contraire des chefs précoloniaux et de l'élite socialiste du FRELIMO de la première heure aurait décidé de "manger seule », sans partager les acquis du pouvoir avec leurs sujets. 
niant à voix haute l'existence de la sorcellerie, l'administrateur, et par extension le gouvernement, par leur propre gestion du pouvoir, parlaient silencieusement le langage de la sorcellerie. Le discours rationaliste explicite et les attaques continues des lions serraient plus fortement les doubles contraintes, exacerbant la crise.

\section{Masques ambigus}

Une vibrante tradition de danse des masques existe en pays makonde, qui ne cesse de se transformer depuis la période coloniale. Souvent décrits comme " masques rituels », les performances des mapiko sont aussi bien un terrain de négociation symbolique et de mise en scène du social ${ }^{27}$. Loin du stéréotype de l'art traditionnel africain qui serait immuable et impersonnel, les transformations sociales et stylistiques de ces «pratiques signifiantes » (Comaroff \& Comaroff 1992: 37) répondent aux sollicitations de l'histoire. Par un travail de l'imagination - autant individuel que s'inscrivant dans des formes et dans des genres transmis et continuellement réinventés - les masques mettent en performance l'histoire et l'identité/(altérité), aussi à travers les chansons qui accompagnent la danse ${ }^{28}$. Le langage, la pratique et les catégories esthétiques des mapiko sont en relation étroite avec ceux de la sorcellerie, par des notions discursives et des techniques du corps liées à la maîtrise, au danger et à la difficulté à déchiffrer ${ }^{29}$. Il existe donc plusieurs raisons pour entendre ce que les masques eurent à dire ou taire, à propos de la crise des lions.

Pendant et après la lutte de libération, la pratique de la danse des masques se transforma radicalement, en incorporant la symbolique ainsi que les contradictions idéologiques de la révolution. À travers la pratique multiforme des mapiko (et aussi à travers les rituels d'initiation) (Israel 2006), la symbolique et l'idéologie du FrELIMo furent inscrites au sein des subjectivités. Un nouveau style de chanson émergea au sein des danses de masques les plus politisées, sous le jour d'un autre dispositif de double contrainte. Les directives culturelles du parti encourageaient les « artistes populaires » à produire un art bien-pensant qui raconte leur vie et leurs souffrances quotidiennes (injonction explicite), contre l'art bourgeois des sentiments individuels et l'expression traditionnelle (obscurantiste) des mystères spirituels

27. Les mapiko sont des danses de masques liées aux rites de puberté masculins des Makonde. Le moment central de ces rituels est la «défiguration » («defacement») (TAUSSIG 1999) du secret du masque par son exposition aux initiés, voir aussi Kingdon (2002: 41 et sq.). Les mapiko sont dansés publiquement, mais l'identité du danseur est un «secret public » (TAUssig 1999) pour les femmes et les non-initiés.

28. Je renvoie à mes propres travaux pour l'histoire des mapiko (IsRAEL 2005, 2008), voir aussi Dias \& Dias (1970) et Kingdon (2002).

29. Étymologiquement, un lipiko est une chose transformée (kupika) ou «fabriquée », comme les lions de Muidumbe (West 2005 : 61-63; IsRAEL 2008). 
Un MASQUe LIPIKO REFIGURANT SAMORA MACHEL, PREMIER PRÉSIDENT DU MOZAMBIQUE

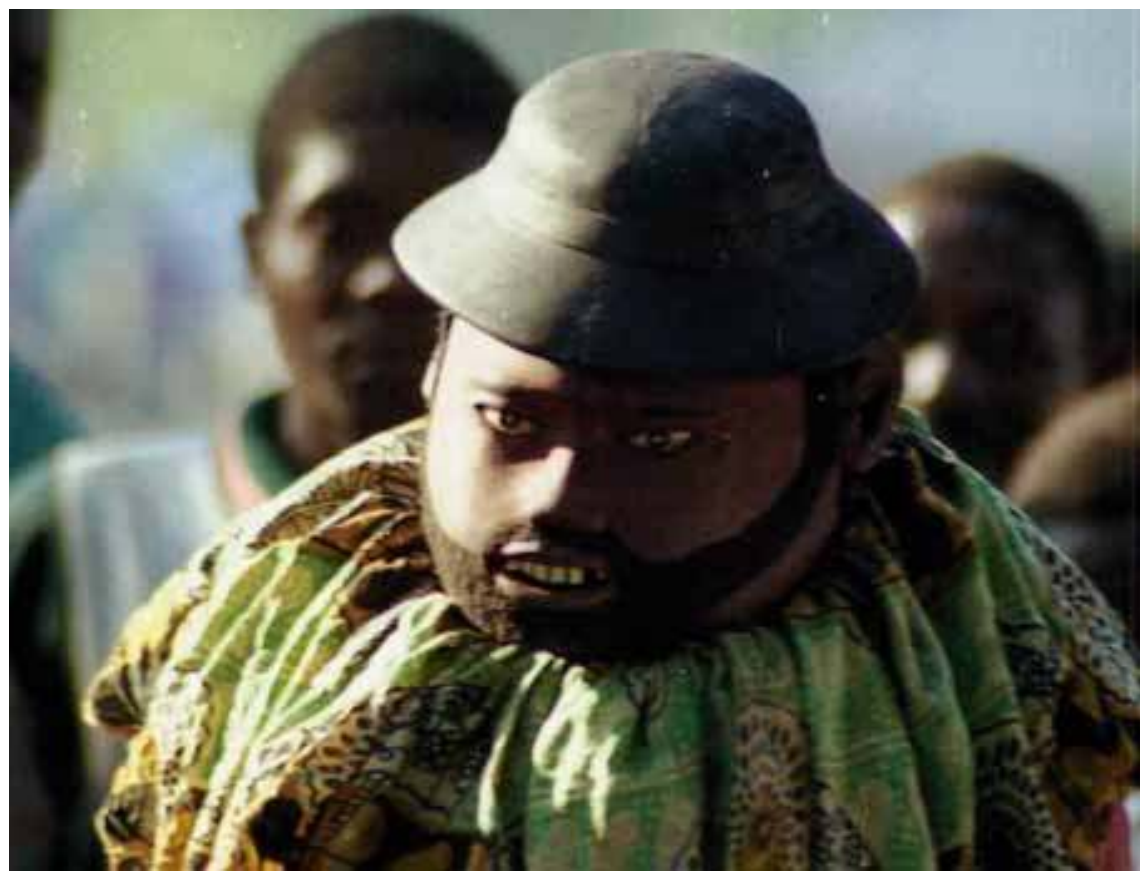

et des provocations entre sexes. Les expériences fondatrices de la nation mozambicaine devaient être au centre de cet art nouveau : l'oppression coloniale, le travail forcé, la lutte de libération... En même temps, le «secret public » (Taussig 1999) du régime socialiste était la répression de la dissension. Sur la place publique, seules devaient être entendues des voix de louange de la révolution (deuxième injonction et menace implicites).

Les deux chansons du groupe de mapiko de Mwambula commentant la crise des lions - chantées sur la place publique - montrent le blocage expressif induit au long terme par ce dispositif de double contrainte.

Mashilikali tuke

Tuke tukavake vantumi

Avana' vantumi, vitannola
Vous, du gouvernement, allons-y

Allons, chassons les lions

Ce ne sont pas des lions, ce sont des vampires

Cette chanson s'ouvre avec une invocation formulaire au gouvernement — trope commun dans la chanson politisée apparue après $1975^{30}$ — invitant

30. Pourtant, le choix du préfixe en $m a$-, forme de pluriel vocatif à la deuxième personne, indique subtilement une distanciation par rapport au gouvernement: vous, du gouvernement. La forme est successivement camouflée par l'utilisation répétée du pluriel à la deuxième personne (tu-), aussi en forme vocative (tuke tukavake). 
à chasser les lions, ce qui implique l'acceptation de la thèse selon laquelle ils seraient des animaux que l'on peut tuer. Le troisième vers dément l'affirmation précédente, en disant qu'il ne s'agit pas de vrais lions, mais de vampires (vitannola).

L'enthousiasme pour la mort du lion n'est pas non plus dénué de contradictions dans le texte suivant, articulé autour des tropes de pleur et de joie de la chanson politique.

Kulila kwatulila tuvamwidumbi wetu

\section{Ntumi mpalakele}

Ntumi mpalakele kwamunjadike

Vanjungu 'ndivashema

Vandyaloka ku Maputo

Ntumi vandimmyaa

Tuvamwidumbi kupuwa

Ntumi mpalakele

Ntumi mpalakele kwamunjadike
On pleure et l'on pleure, nous, les gens de Muidumbe

Ramenez le lion

Ramenez le lion là où vous l'avez sorti On a appelé des Blancs

Ils sont venus de Maputo

Ils ont tué le lion

Nous, de Muidumbe, nous fêtons

Ramenez le lion

Ramenez le lion là où vous l'avez sorti

Le lion doit être «ramené » d'où il a été «sorti »: donc il n'est pas une bête sauvage, mais un animal fabriqué. Pourtant, il a été bien tué par des Blancs venant de Maputo, pour le bonheur des gens de Muidumbe.

\section{LE MASQUe NA-UPANGa MENACE LA FOULE AVEC SA MACHETTE}

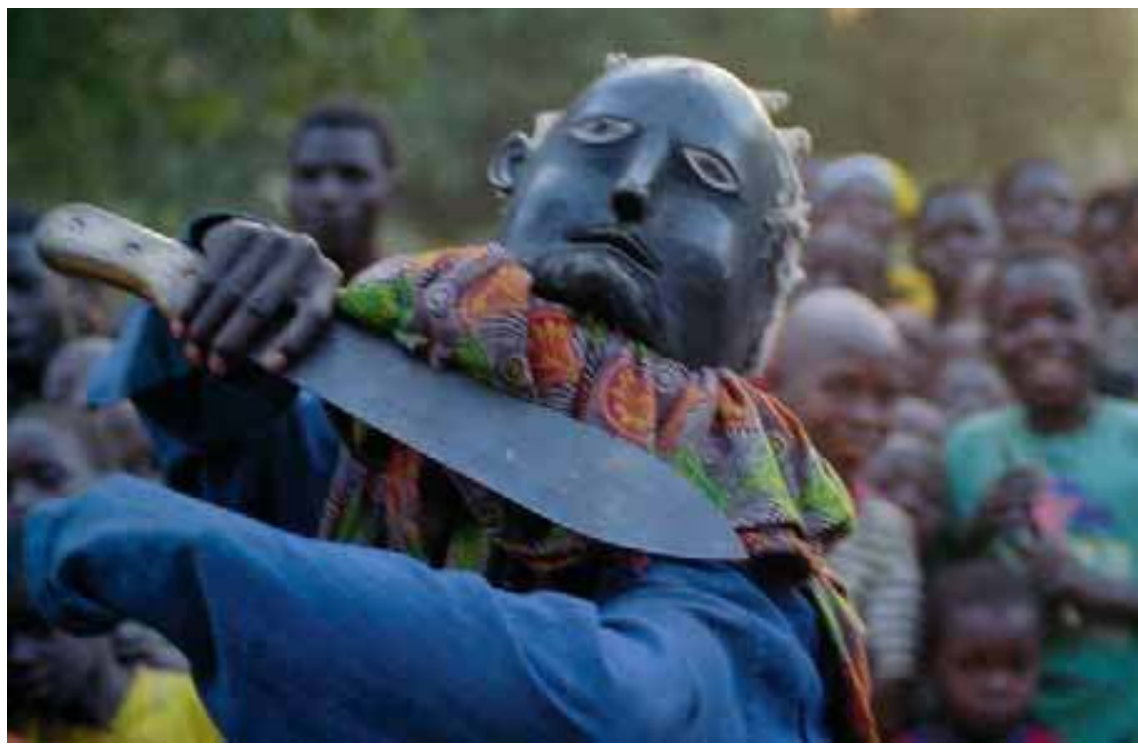

En 2001, un groupe de jeunes hommes du village de Mwatide inventa un nouveau masque qui, en peu de temps, se répandit sur tout le plateau makonde, devenant la danse de masque générationnelle des plus jeunes. Ces 
mapiko, appelés na-upanga (avec-une-machette), s'inspiraient des formes récentes de danses de masques juvéniles, puisés dans une tradition de mascarades de critique sociale des plaines du Messalo, dont l'une des caractéristiques est la mise en scène moralisante mais pleine d'ambiguïté des activités des sorciers.

Sous une forme certainement abstraite, symbolique et métaphorique, na-upanga avait mis en scène la structure des événements de la crise de Muidumbe quelques temps avant qu'ils n'arrivent. Le masque na-upanga représente un ancien (nang'olo) aux cheveux blancs et à l'air sérieux. Pourtant, il s'habille comme un jeune homme «sans respect» ("akavé ishima ») et partage les prédilections culturelles globalisées de la jeunesse des villages : kung-fu, danse moderne, acrobaties à vélo, football. Il se promène armé d'une machette, avec laquelle il menace la foule : «Crains na-upanga, il va te tuer ! » Il s'en prend particulièrement aux sorciers : « Si tu danses la sorcellerie, la terre va t'engloutir. »

Quelque temps après la fin de la crise (en juillet 2003), na-upanga en proposa un commentaire visuel plus explicite. Un nouveau sketch avait été ajouté à la danse : les choristes du groupe se promenaient sur le terrain de danse, en levant le bras de façon menaçante. Ils chantaient une chanson de provocation électorale (contre la RENAMO), adaptée pour l'occasion :

Woya-woyana woya-woyana

Kubondi akó

Ukatauka mmudye Shavimbi mwatutandale'

Avalá !

'Navaleke!
C'est le coupe-coupe, tue-tue

Là-bas, dans les plaines

$\mathrm{Si}$ vous insistez, demandez à Savimbi comment on fait ${ }^{31}$

Ceux-là !

$\mathrm{Ne}$ les laissez pas!

Ensuite, ils chantaient en chœur vashikaji noma, l'hymne des lyncheurs.

Ce petit refrain était apparu comme chanson de provocation utilisée dans les compétitions de football entre équipes villageoises. Il s'agit de deux mots kiswahili : «vashikaji» («jeunes ») et le mot noma, qui a pris localement une signification spéciale et qui est traduit en portugais mozambicain (PM) par l'expression «matreco ». En portugais, un matreco désigne littéralement un pantin, une figurine du jeu de «babyfoot». En portugais mozambicain, ce mot est utilisé pour qualifier une personne privée d'initiative et de volonté, qui se laisserait manipuler facilement. Le verbe dérivatif «matrecar» peut signifier arnaquer quelqu'un ou bien asservir la volonté de quelqu'un à son propre désir ${ }^{32}$. En shimakonde, on dit qu'un matreco est «comme un lindandosha», un mort-vivant privé de volonté par un sorcier qui utilise son corps à ses propres fins (West 2005 : 186-188, 286 n. 22). Dès le début

31. La figure de Savimbi est lue comme une contrepartie angolaise de Dhlakama, le leader de la RenAmo, et la nouvelle de sa mort retentit au pays makonde.

32. Le verbe a aussi d'autres connotations : (1) s'habiller mal, (2) manquer au compromis ou à la parole donnée, (3) déprécier, (4) humilier (LOPES et al. 2002: 101). 
de la crise, ce chœur de football fut utilisé par les lyncheurs pour accompagner les «lions » à leur mort. Qu'ils fussent jeunes ou vieux, il s'agissait toujours de «jeunes pantins »: privés de volonté, sans initiative, comme des morts-vivants au service d'un sorcier.

\section{« Jeunes pantins»: nostalgie et envie d'agency}

En novembre 2003, le gouvernement de la province envoya une autre brigade armée à Muidumbe, cette fois-ci avec l'ordre d'arrêter les responsables de l'émeute de Pâques. Cinq jeunes hommes furent capturés. Celui qui était monté sur le podium pour défier l'autorité du gouvernement local fut ensuite (en juillet 2004) condamné à la prison à vie, accusé de sédition et d'homicide. Par la suite, certains des parents des victimes intentèrent des actions judiciaires contre les présidents des villages ayant protégé ou encouragé les lynchages. Certains d'entre eux furent arrêtés, mais aucun ne fut condamné à la prison.

Il est très difficile d'esquisser une cartographie sociale des lynchages. En l'observant à la loupe, la crise se décompose et perd son unité, laissant place à une multiplicité de situations et de logiques singulières, les lynchages obéissant parfois à des logiques familiales, parfois à des conflits de pouvoir, parfois à des inimitiés personnelles, parfois au simple hasard. La diffusion de la rumeur et de la peur dépassa toute barrière sociale. En revanche, chaque lynchage eut son histoire spécifique, tant en ce qui concerne le profil social des bourreaux et des victimes, que les modalités d'exécution.

Cela dit, la jeunesse eut un rôle prépondérant dans la constitution des foules chassant les sorciers. Les lynchages publics, menés dans les villages communaux du plateau, étaient toujours devancés par des groupes de jeunes hommes, qualifiés parfois comme «jeunes du bazar» («vanemba vakubazari »). Cette expression indique des jeunes adolescents oisifs, qui «vaquent» dans les bazars de village, en attente d'une quelconque opportunité de travail $^{33}$. Certains d'entre eux arrivent à amasser des petites fortunes : il existe des histoires de «self-made men » qui commencent par vendre des cigarettes et finissent par posséder un petit magasin. La plupart d'entre eux, pourtant, sont voués à l'échec, souvent à l'alcoolisme et au crime mineur, parfois au suicide.

Ailleurs en Afrique, le rôle prépondérant de la jeunesse dans les chasses au sorcier (Comaroff \& Comaroff 1999 : 287-290 ; Ashforth 2005 : 256257 ; Niehaus 1998) a été mis en relation avec les délabrements sociaux

33. Depuis la fin de la guerre civile, des petits marchés régis par l'économie informelle ont surgi un peu partout dans les grands villages communaux de Muidumbe. Les magasins présents dans ces marchés sont gérés pour la plupart par des jeunes entrepreneurs, achetant des produits en Tanzanie ou dans la province de Nampula. La concurrence est pourtant très dure, le marché restreint, et la protection politique parfois nécessaire. 
provoqués par le capitalisme sauvage (Comaroff \& Comaroff 2001). Il est sans doute pertinent de lire le malaise de cette jeunesse comme une forme du syndrome généralisé d'impuissance et d'émasculation symbolique induit, notamment en Afrique, par l'âpreté des politiques néo-libérales (Comaroff \& Comaroff 2000; Mbembe 2000b : 107-113). L'ouverture des marchés ainsi que celle de l'imaginaire de la consommation renvoient les jeunes de Muidumbe à leur position marginale, au manque d'opportunités et finalement à un futur de désespoir. La rage et le malaise des jeunes lyncheurs de Muidumbe peuvent toutefois se lire aussi sous un autre jour, en prenant en compte des facteurs plus complexes qui touchent à l'histoire de leurs subjectivités, et notamment aux contradictions induites par les doubles contraintes idéologiques de la révolution du FreLimo.

John Mowitt (2002 : 51-53) montre comment le débat théorique sur la notion d'agency dans certains courants du postmarxisme a été marqué par des notions nostalgiques. La revendication théorique du sujet comme porteur d'agency viserait autant à justifier la possibilité de toute action politique, qu'elle véhiculerait une nostalgie d'expériences d'engagement et de dissidence $^{34}$. La critique théorétique de Mowitt est pertinente aussi pour lire le discours politique du Frelimo, fondé sur une idéologie nostalgique de l'agency ${ }^{35}$. La lutte de libération nationale y apparaît comme l'expérience fondatrice qui montre comment la volonté collective révolutionnaire est capable de briser tout joug et toute contrainte. Depuis, toute action politique à l'époque postcoloniale se mesurera (nostalgiquement) au mythe héroïque de la lutte armée. Tout échec est imputé à un défaut ou à un excès d'agency : le peuple qui n'est pas suffisamment révolutionnaire, l'ennemi du peuple qui complote en secret. Toute victoire est redevable de l'agency du guide du peuple, le parti. Le manichéisme révolutionnaire s'articule autour de notions d'agency: le peuple est désormais une entité homogène, divisé entre ceux qui veulent et ceux qui ne veulent pas (la révolution, l'effort, la lutte, le progrès, le socialisme...). Comme le montre Geffray (1990) les facteurs structurels — notamment socioculturels — sont sous-estimés ou méconnus

34. « Though I by no means wish to disparage the experience of political dissidence that is clearly inscribed within such a position, it is hard not to conclude that this otherwise rigorous engagement with Althusser ends in what I have called nostalgia. The subject must be restored, because without one - even an impossible one - resistance is unthinkable and therefore futile. It is in this sense that I perceive the issue of agency to be at stake in this debate within post-Marxism, and furthermore that I feel entitled to kvetch about the way agency operates in the discourse of identity politics » (MOwITT 2002: 53). Pour une critique de notions nostalgiques ou identitaires de l'agency inspirée à Mowitt, voir LALU (2000).

35. Je remercie Premesh Lalu pour cette suggestion. J'utilise «agency » dans le premier des deux sens esquissés par ORTNER (2001: 78) : «In probably the most common usage, "agency" is virtually synonymous with the forms of power people have at their disposal, their ability to act on their own behalf, influence other people and events, and maintain some kind of control in their own lives », voir aussi AHEARN (2001). 
dans les analyses politiques du Frelimo $^{36}$. La page blanche ne peut être écrite que par un effort héroïque de la volonté (Mbembe 2000a : 20).

Ces notions nostalgiques de volontarisme et résistance (agency) furent inscrites au cœur des subjectivités de la génération qui lutta pour l'indépendance. En pays makonde, elles prennent des formes aussi fortes que fut la participation à la lutte. Une génération entière d'hommes et de femmes embrassa les armes pour lutter pour sa propre autodétermination. Ces jeunes étaient autorisés à briser — au nom de la révolution — le respect pour leurs aînés. Significativement et symboliquement, l'histoire mythique de l'un des leaders de la guérilla en pays makonde commence par le meurtre du père, allié avec les Portugais. Les «faiseurs de guerre » («vanantenda ing'ondo ») en pays makonde furent autant embrigadés et idéologisés, qu'ils acquirent un sens démesuré de leur puissance. Aux yeux de la nation entière, ils n'étaient plus un groupe méconnu de «primitifs », mais le peuple guerrier qui avait pris les armes, les pionniers de l'homme nouveau dans les « zones libérées » («zonas libertadas »). Le gouvernement repaya la fidélité par un programme extensif de pensions de guerre. Localement, des nouvelles hiérarchies sociales basées sur le capital symbolique accumulé pendant la participation à la guerre se dressèrent.

La première génération des fils de la révolution, les «enfants de Mondlane » nés pendant la guerre, put profiter du nouveau système scolaire mis en place par le Frelimo dans les zones libérées (Mazula 1995). Les générations suivantes - la plupart de leurs représentants au moins — n'eurent aucune part du capital symbolique et social dérivé de la lutte. Les structures s'effondrèrent à cause de la guerre et seuls l'idéologie et le parti survécurent. Ces jeunes, élevés dans un bain de nostalgie, étaient condamnés à vénérer les symboles de l'agency de leurs pères (monuments, drapeaux, sièges du parti), sans pour autant en bénéficier. Éduqués dans la haine de la RENAMO, non seulement par leurs pères mais par les atrocités commises autour du plateau par la RENAMO elle-même en 1990-1991, ils n'avaient aucune possibilité d'articuler une voix de dissension politique.

En guise de conclusion, j'essaie d'écouter, de regarder le masque naupanga. Que dit ce jeune homme au masque de vieux (nang'olo: ancien, puissant), qui brandit une machette et s'en prend aux sorciers ? À mon sens, il met en scène la même impasse qui amena les jeunes aux explosions de violence et aux lynchages. Il représente (ante litteram) les actions de jeunes

36. On peut mesurer la différence avec le paradigme du développement, qui mesure tout échec et toute réussite sur des facteurs structuraux (notamment déclinés dans un jargon socio-économique), et qui, par l'effacement systématique de l'agency, aboutit à la dépolitisation des sociétés dans lesquelles il opère (FERGUSON 1990). 
lyncheurs qui auraient défié leurs aînés, mus par un désir refoulé et inconfessable de saisir leurs places et leur capacité d'action (agency). En mettant un masque de vieux et en brandissant une machette, ces lyncheurs voyaient dans les sorciers des miroirs d'eux-mêmes: des «jeunes pantins » privés de capacité d'action et de volonté. L'un des joueurs du groupe de na-upanga me dit, souriant d'un air ironique : "Wetu tuvanemba, tuvashikaji noma, yadao... » (« Nous les gars, nous sommes de "jeunes pantins", nous aussi... »).

L'envie (ing'ou) ${ }^{37}$ est souvent invoquée comme moteur de toute action de sorcellerie (West 2005 : 46). Les lyncheurs étaient aussi bien pris dans une sorte d'envie d'agency, dérivée de leur paralysie sociale et idéologique. Tout en étant dirigée contre les sorciers, son objet était (par personne interposée) les aînés — et le système idéologique paradoxal qu'ils représentaient (sans l'avoir pourtant produit). En elle-même, elle était paradoxale, appelant à la fois à un retour à l'ordre («nous sommes le FrELIMo!») et à une subversion de l'ordre idéologique (par les inversions symboliques). Chez ces jeunes - qui un jour confessaient avec orgueil avoir participé aux lynchages («Pourquoi pas? Il faut faire quelque chose ! ») et qui le jour après niaient tout engagement («Moi ? J'ai juste regardé ») — cette envie d'agency ne savait pas s'exprimer autrement que par un défoulement violent. Certes, seulement certains lynchèrent. Il y a pourtant des raisons (historiques, sociales, ethnographiques) pour défendre que cette structuration nostalgique/envieuse du champ idéologique de l'agency agisse — en des modalités et intensités différentes, et avec des résultats fort divergents - sur une génération entière de jeunes hommes de Muidumbe.

La crise de Muidumbe expose - entre autres - des tensions et contradictions latentes, qui ont à faire avec des dispositifs disciplinaires de doubles contraintes inscrits dans les subjectivités, pendant le processus de vernacularisation du projet révolutionnaire de nation du FRELIMO. L'intervention idéologique du gouvernement - renvoyant le «peuple » à son analphabétisme et à son caractère «ethnique » et de par ce fait le blâmant de la crise par une imputation de manque d'agency révolutionnaire - tendait structurellement à serrer davantage ces doubles contraintes et à dramatiser leurs effets.

Certes, les rumeurs globales eurent un poids dans l'exacerbation de la paranoïa et de la violence. Dans la crise de Muidumbe se retrouvent des éléments qui l'inscrivent dans des dynamiques plus larges: les rumeurs concernant zombies, vampires et le trafic d'organes; l'émergence de nouveaux paradigmes locaux de la sorcellerie ; les clivages générationnels mettant en exergue le «malaise d'émasculation» des jeunes générations dans le contexte néo-libéral. Le malaise des «jeunes pantins » de Muidumbe ne

37. Au sens latin de invidia: le sentiment de malveillance face aux succès d'autrui. Selon la définition de George Foster (1972: 168), l'envie (envy) découle du désir d'acquérir quelque chose qu'autrui possède alors que la jalousie s'enracine dans la peur de perdre ce que l'on possède déjà (je remercie un lecteur anonyme pour cette suggestion). 


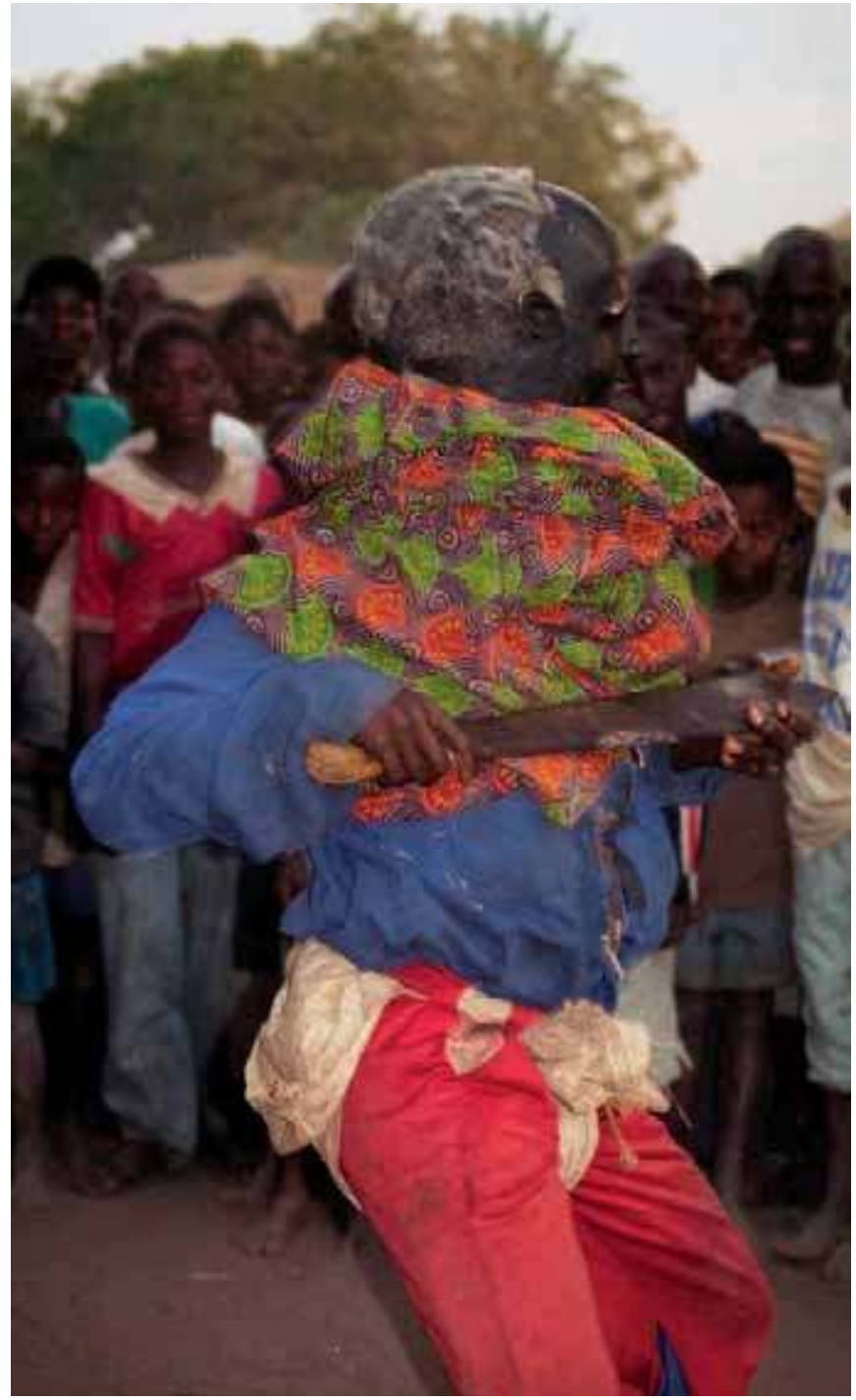

peut pourtant pas être compris hors d'une histoire de la subjectivation (projet esquissé dans Mbembe 2000a : 16-17). Leur « envie d'agency » serait autant induite par l'impuissance économique que par la structuration paradoxale et nostalgique du capital symbolique que leurs aînés accumulèrent lors de la lutte de libération nationale. La sorcellerie et la danse des masques s'attachent, par des moyens différents, à mettre en scène l'insolubilité apparente de ces paradoxes.

Centre d'études africaines, EHESS, Paris ; Centre for Humanities Research, University of the Western Cape, Bellville, South Africa. 


\section{BIBLIOGRAPHIE}

ADAM, Y.

1993 « Mueda, 1917 : Resistência, Colonialismo, Libertação e Desenvolvimento », Arquivo, Boletim Do Arquivo Histórico De Moçambique, 14 : 4-102.

Ahearn, L.

2001 «Language and Agency », Annual Review of Anthropology, 30 : 109-137.

AshForth, A.

2005 Witchcraft, Violence and Democracy in South Africa, Chicago-Londres, Chicago University Press.

AsKew, K.

2002 Performing the Nation: Swahili Music and Cultural Politics in Tanzania, Chicago, University of Chicago Press.

BATESON, G.

1977 Vers une écologie de l'esprit, t. 1, Paris, Éditions du Seuil.

2000 Steps to an Ecology of Mind, Chicago-London, University of Chicago Press.

BAYART, J.-F.

1990 L'État en Afrique. La politique du ventre, Paris, Fayard.

BuRgess, T.

2005 «Introduction to Youth and Citizenship in East Africa », Africa Today (Special Issue) : VII-XXIV.

BUTLER, J.

1997 The Psychic Life of Power, Stanford, Stanford University Press.

CAHEN, M.

1987 Mozambique, la révolution implosée. Études sur douze années d'indépendance (1975-1987), Paris, L'Harmattan.

2000 «The Mueda Case and Maconde Political Ethnicity. Some Notes on a Work in Progress », Africana Studia (Porto), 2 : 29-46.

Comaroff, J. \& Comaroff, J.

1992 Ethnography and the Historical Imagination, Boulder-San Francisco-Oxford, Westview Press.

1993 Modernity and Its Malcontents. Ritual and Power in Postcolonial Africa, Chicago, The University of Chicago Press.

1999 «Occult Economies and the Violence of Abstraction : Notes from the South African Postcolony », American Ethnologist, 26 (2) : 279-303.

2000 «Réflexions sur la jeunesse. Du passé à la postcolonie », Politique africaine, 80: 90-110.

2001 Millenial Capitalism and the Culture of Neoliberalism, Durham, Duke University Press.

2002 «Alien-Nation: Zombies, Immigrants, and Millennial Capitalism», The South Atlantic Quarterly, 101 (4) : 779-805. 
Condominas, G.

1980 L'espace social à propos de l'Asie du Sud-Est, Paris, Flammarion.

Dias, J. A. \& DiAs, M.

1970 Os Macondes de Moçambique, vol. III : Vita social e ritual, Lisboa, Junta de Investigações do Ultramar.

FERGUSON, J.

1990 The Anti-Politics Machine. "Development», Depoliticization, and Bureaucratic Power in Lesotho, Cambridge-London, Cambridge University Press.

FOSTER, G.

1972 « The Anatomy of Envy: A Study in Symbolic Behavior », Current Anthropology, 13 (2) : 165-202.

GeFFray, C.

1988 «Fragments d'un discours du pouvoir (1975-1985)», Politique africaine, $29: 71-85$.

1990 La cause des armes au Mozambique: anthropologie d'une guerre civile, Paris, Karthala.

GESCHIERE, P.

1995 Sorcellerie et politique en Afrique. La viande des autres, Paris, Karthala.

2000 «Sorcellerie et modernité : retour sur une étrange complicité », Politique africaine, $79: 17-32$.

Hall, M. \& Young, T.

1997 Confronting Leviathan, Mozambique since Independence, London, Hurst \& Co.

ISRAEL, $\mathrm{P}$.

2005 «Mapiko Masquerades of the Makonde : Performance and Historicity », in H. Arero \& Z. Kingdon (eds.), Eastern African Contours : Reviewing Creativity and Visual Culture, London, Horniman Museum : 99-121.

2006 «"Kummwangalela Guebuza". The Mozambican General Elections of 2004 in Muidumbe and the Roots of the Loyalty of Makonde People to FreLIMO », Lusotopie, XIII (2) : 103-125.

2008 Masques en transformation. Performances mapiko des Makonde (Mozambique) : historicité, création et révolution, Thèse de $3^{\mathrm{e}}$ cycle, Paris, EHESS.

À paraître «The "War of the Lions". Man-eating Lions and Witch-hunts in Muidumbe (Mozambique), 2002-2003 », Journal of Southern African Studies.

JACKSON, M.

1990 «The Man Who Could Turn into an Elephant: Shape-shifting among the Kuranko of Sierra Leone », in M. JACKSON \& I. KARP (eds.), Personhood and Agency, The Experience of Self and Other in African Cultures, Uppsala, Smithsonian Institution Press : 59-78.

Joset, P. E.

1955 Les sociétés secrètes des hommes-léopards en Afrique Noire, Paris, Payot. 
KAPFERER, B.

1997 The Feast of the Sorcer. Practices of Consciousness and Power, Chicago, The University of Chicago Press.

KingDON, Z.

2002 A Host of Devils. The History and Context of the Making of Makonde Spirit Sculpture, London-New York, Routledge.

LALU, P.

2000 «The Grammar of Domination and the Subjection of Agency: Colonial Texts and Modes of Evidence », History and Theory, 39 (4) : 45-68.

Lімвомво, O.

2003 «Leões de Muidumbe», in Questão de Fundo, Pemba, Radio Moçambique.

Lopes, A. J., Sitoe, S. J. \& Nhamuende, P. J.

2002 Moçambicanismos. Para um Léxico de Usos do Português Moçambicano, Maputo, Livraria Universitaria, Universidade Eduardo Mondlane.

MAZULA, B.

1995 Educação, Cultura e Ideologia em Moçambique : 1975-1985, Lisboa, Edições Afrontamento.

Mbembe, A.

2000a « À propos des écritures africaines de soi », Politique africaine, 77 : 16-44.

2000b De la postcolonie. Essai sur l'imagination politique dans l'Afrique contemporaine, Paris, Karthala («Les Afriques»).

2005 Pour l'avant-propos à la deuxième édition.

MoORe, H. \& SANDERs, T.

2001 «Introduction» in H. L. Moore \& T. SANDERs (eds.), Magical Interpretations, Material Realities. Modernity, Witchcraft and the Occult in Postcolonial Africa, London, Routledge.

MowitT, J.

2002 Percussion : Drumming, Beating, Striking, Durham-London, Duke University Press.

Nсомо, B. L.

2003 Uria Simango, um homem, uma causa, Maputo, Novafrica.

NieHAUS, I. A.

1998 «The ANC Dilemma: The Symbolic Politics of Three Witch-Hunts in the South African Lowveld, 1990-1995 », African Studies Review, 41 (3) : 93-118.

NWAKA, G. I.

1986 «The "Leopard" Killings of Southern Annang, Nigeria, 1943-1948 » Africa, 56 (4) : 417-440. 
ORTNER, S. B.

2001 «Specifying Agency: The Comaroffs and their Critics », Interventions, The International Journal of Post-Colonial Studies, 3 (1) : 76-84.

Rich, J.

2001 «"Leopard Men", Slaves, and Social Conflict in Libreville (Gabon), c. 1860$1879 »$, The International Journal of African Historical Studies, 34 (3) : 619-638.

RICEUR, P.

1985 Temps et récit, t. III : Le temps raconté, Paris, Éditions du Seuil.

SANDERS, T.

2004 «Reconsidering Witchcraft: Postcolonial Africa and Analytic (un)Certainties », American Anthropologist, 105 (2) : 338-352.

SCHEPER-Hugues, N.

1996 «Theft of Life : The Globalization of Organ Stealing Rumours », Anthropology Today, 12 (3) : 3-11.

2000 «The Global Traffic in Organs », Current Anthropology, 41 (2) : 191-224.

SERRA, C.

2003a Combates pela mentalidade sociologica, Maputo, Imprensa Universitária.

2003b Cólera e catarse, Maputo, Imprensa Universitária.

SHAw, R.

1997 «The Production of Witchcraft/witchcraft as Production : Memory, Modernity, and the Slave Trade in Sierra Leone », American Ethnologist, 24 (4) : 856-876.

TAUSSIG, M.

1999 Defacement. Public Secrecy and the Labor of the Negative, California, Stanford University Press.

WALKER, R.

1989 «Marxism-Leninism as Discourse : The Politics of the Empty Signifier and the Double Bind », British Journal of Political Science, 19 (2) : 161-189.

West, H. G.

1998 " "This Neighbour is not My Uncle": Changing Relations of Power and Authority on the Mueda Plateau », Journal of Southern African Studies, 24 (1) : 141-160.

2004 «Villains, Victims, or Makonde in the Making ? Reading the Explorer Henry O'Neill and Listening to the Headman Lishehe », Ethnohistory, LI (1) : 1-43.

2005 Kupikilula, Governance and the Invisible Realm in Mozambique, LondonChicago, Chicago University Press.

2007 Ethnographic Sorcery, Chicago-London, Chicago University Press.

À paraître «Govern Yourselves! Democracy and Carnage in Northern Mozambique », in J. Paley (ed.), Toward an Anthropology of Democracy, Santa Fe, School for Advanced Research Press. 
White, L.

1993 «Cars out of Place: Vampires, Technology, and Labor in East and Central Africa », Representations, $43: 27-50$.

1997 «The Traffic in Heads : Bodies, Borders and the Articulation of Regional Histories », Journal of Southern African Studies, 23 (2) : 325-338.

2000 Speaking with Vampires : Rumour and History in Colonial Africa, LondonBerkeley, University of California Press.

\section{RÉSUMÉ}

En 2002-2003, une crise liée à des accusations de sorcellerie eut lieu à Muidumbe, district du nord du Mozambique et berceau de la lutte de libération nationale. Des lions attaquaient et dévoraient les paysans dans les zones de production agricole, chaque nouvelle d'une attaque déchaînant une chasse au sorcier meurtrière dans les villages " communaux». Les rumeurs qui alimentaient la violence concernaient notamment l'existence d'une société secrète - sur le mode de celles documentées ailleurs en Afrique pendant la période coloniale —, se déguisant à l'aide de peaux et pattes et tuant pour enlever et revendre des organes. La crise donna lieu à une ébauche d'émeute politique, dans laquelle des jeunes s'en prirent à l'ordre constitué, mettant en scène des inversions symboliques. Il est possible de relier cette crise à des phénomènes sociaux plus larges, telles que les rumeurs globales concernant zombies, vampires et le trafic d'organes; l'émergence de nouveaux paradigmes locaux de la sorcellerie; le "malaise d'émasculation» des jeunes générations dans le contexte néo-libéral. Dans la lecture que je fais de cette crise, elle renvoie à l'articulation locale du projet révolutionnaire de construction de la nation socialiste mozambicaine, à la formation historique des subjectivités de ses pionniers, et notamment à l'incorporation de dispositifs idéologiques paradoxaux.

\section{ABSTRACT}

Lacerations and Rumours. Witch-hunting and the Ideological Legacy of the Socialist Revolution in Mozambique (Muidumbe, 2002-2003). - In 2002-2003, a crisis connected to accusations of sorcery took place in Muidumbe, a rural district of Northern Mozambique, being the cradle of the Struggle for Liberation. As lions attacked and devoured people in the zones of agricultural production, the news of the killings unleashed deadly witch-hunts in the "communal villages" of the district. The violence was fuelled by rumours concerning the existence of a secret society (similar to those acting elsewhere in Africa in the colonial era) whose members disguised themselves with lion skins and paws, and killed in order to obtain and sell organs. The crisis culminated with a political uprising, in which the young people defied the social order through witch-killings and symbolic inversions. This crisis can be connected to broader social phenomena, such as global rumours concerning zombies, vampires and the traffic of organs, the emergence of new local paradigms of sorcery, the "syndrome of emasculation" of African youth in the neo-liberal context. 
In this paper, I read this crisis as pointing to the local articulation of the revolutionary project of nation building in Mozambique, to the historical process of subjectivation of its pioneers, and specifically to the embodiment of double-binding ideologies.

Mots-clés/Keywords: Mozambique, Muidumbe, agency, masques, postsocialisme, rumeurs, subjectivation/Mozambique, Muidumbe, agency, masquerade, post-socialism, rumours, subjectivation. 